\title{
Intragastric Feeding in Type I Glycogen Storage Disease: Factors Affecting the Control of Lactic Acidemia
}

\author{
CHARLES A. STANLEY, ${ }^{(18)}$ JAMES L. MILLS, AND LESTER BAKER \\ Division of Endocrinology/Diabetes, Department of Pediatrics, University of Pennsylvania School of Medicine and the \\ Children's Hospital of Philadelphia, Philadelphia, Pennsylvania, USA
}

\section{Summary}

Continuous nocturnal intragastric feeding, combined with frequent daytime feedings, has been reported to improve both linear growth and the metabolic abnormalities in patients with glucose6-phosphatase deficiency (Type I Glycogen Storage Disease). However, elevated blood levels of lactate have persisted. The present studies explore the relationship between blood lactate concentrations in six patients with glucose-6-phosphatase deficiency and variations in the rate and composition of the intragastric feeding. Blood lactate and plasma glucose concentrations were determined at rates of dextrose administration ranging from 3-34 $\mathrm{mg} / \mathrm{kg} / \mathrm{min}$. Dextrose infusion at $100-200 \%$ of estimated normal glucose production rates gave the best control of blood lactate concentrations. Lower rates of dextrose infusion resulted in significantly higher blood lactate levels; higher rates produced hyperglycemia, but no significant further reduction of blood lactate. At identical rates of glucose administration, a dextrose-containing infant formula and a high carbohydrate enteric feeding solution gave no significant improvement in control of blood lactate levels compared to dextrose alone. Plasma glucose levels fell more rapidly when intragastric feeding was stopped than after a mixed meal and hypoglycemia appeared to develop before counter-regulatory responses could be mobilized. These observations may account for the increased susceptibility to symptomatic hypoglycemia reported in patients treated with intragastric feeding.

\section{Speculation}

These results indicate that intragastric glucose infusion at a rate equal to or slightly greater than normal hepatic glucose production gives maximal control of the metabolic abnormalities in patients with glucose-6-phosphatase deficiency. Complete normalization of hyperlactatemia and hypertriglyceridemia in these patients may require the development of additional forms of therapy.

Patients with a deficiency of hepatic glucose-6-phosphatase activity (Type I Glycogen Storage Disease) have severe growth retardation. Other manifestations of this disorder include hypoglycemia, lactic acidosis, elevations of plasma triglyceride and uric acid concentrations, osteoporosis and a bleeding tendency (11). The absence of glucose-6-phosphatase activity means that the liver cannot produce glucose from glycogen stores or by gluconeogenesis. Plasma glucose concentrations cannot, therefore, be maintained in a normal range in the postabsorptive state and recent efforts to treat these patients have been directed at prevention of the hypoglycemia (12). Burr et al. (3) introduced the concept of using continuous intragastric feeding to manage this disorder in 1974. A number of workers have reported that continuous nocturnal intragastric feeding, combined with frequent daytime feedings, improves both growth and the metabolic abnormalities in infants and children with glucose-6-phosphatase deficiency $(4,5,7,8,9)$.

Our own experience with the nocturnal intragastric feeding regimen has been consistent with the improvement in growth reported by others. However, elevated levels of blood lactate have persisted. The present studies were, therefore, undertaken to explore the relationship between concentrations of blood lactate in patients with glucose-6-phosphatase deficiency and variations in the rate of administration or composition of the intragastric feeding. In addition, the response to acute termination of intragastric infusion was examined, since it has been reported $(15,13)$ that patients with this regimen may develop severe symptoms of hypoglycemia at levels of plasma glucose which had previously been well tolerated.

\section{MATERIALS AND METHODS}

Six patients with biopsy-documented glucose-6-phosphatase deficiency were included in these studies. All patients were on a fructose and galactose-free diet with frequent feedings during the day and, at home, received continuous intragastric $20 \%$ dextrose overnight from 8 P.M. to 8 A.M. using a portable infusion pump (model 911, Extracorporeal). The rate of dextrose infusion was approximately $10 \mathrm{mg} / \mathrm{kg} / \mathrm{min}$. Dietary instructions did not prescribe quantities of food during the day, but advised the use of high carbohydrate snacks between meals, including a dextrosecontaining milk substitute (Cho-Free) and a fruit flavored drink (Kool-aid) made with dextrose. More recently, a need to specify carbohydrate intakes equal to $7-10 \mathrm{mg} / \mathrm{kg} / \mathrm{min}$ of glucose during the day has been appreciated (see Results). Table l shows the age and duration of treatment of these patients. Patients 1 and 3 had feeding gastrostomies while the other four passed a naso-gastric tube each night. Patients 4 and 5 were siblings.

For the studies reported here, patients were admitted to the Clinical Research Center at the Children's Hospital of Philadelphia. Venous blood samples were drawn from indwelling needles kept patent by flushing with heparinized saline. Plasma glucose levels were immediately determined by the glucose-oxidase method using a Beckman glucose analyzer. Blood lactate and pyruvate and plasma free fatty acids (FFA) and beta-hydroxybutyrate (BOB) were determined as previously described (16). Other assays were carried out in the hospital clinical laboratories by standard techniques.

For studies on the relationship between the composition of the intragastric infusate and circulating levels of glucose and lactate, blood was obtained at $2-4 \mathrm{~h}$ intervals beginning no earlier than 2 $\mathrm{hr}$ after commencement of the infusion. Infusion flow varied from $2-4 \mathrm{cc} / \mathrm{kg} / \mathrm{h}$ to provide the desired rate of substrate delivery. In these short-term studies, constant rates of intragastric feedings were maintained for 8-16 h. Different rates of infusion were tried on different nights of the patients' hospitalizations. Patients were studied both before the initiation of therapy (patients $4,5,6$ ) and after months to years of overnight intragastric feeding (all patients). The duration of treatment with overnight intragastric 
feedings had no apparent effect on the levels of blood lactate, which were found during different rates of intragastric infusions, in these patients. In studies to compare dextrose alone with nutritionally more complete solutions, a lactose-free milk substitute (Cho-Free, Syntex) with added dextrose and an enteric feeding solution (Vivonex, Eaton) were used. The Cho-Free solution contained $6.4 \%$ carbohydrate (dextrose), $3.5 \%$ fat, and $1.8 \%$ protein (20 cal/oz). The Vivonex contained $23 \%$ carbohydrate (glucose oligosaccharides), $0.145 \%$ fat, and $0.32 \%$ amino acid nitrogen (1 $\mathrm{cal} / \mathrm{cc}$ ). Normal glucose production rates were estimated from the data of Bier, et al. (1) as $\mathrm{Y}(\mathrm{mg} / \mathrm{min})=0.0014 \mathrm{X}^{3}-0.214 \mathrm{X}^{2}-$ $10.411 \mathrm{X}-9.084$, where $\mathrm{X}$ is body weight in $\mathrm{kg}$.

These studies were reviewed and approved by the Committee for Protection of Human Subjects of the Children's Hospital of Philadelphia. Informed consent for the studies was obtained from the parents of the patients.

\section{RESULTS}

\section{EFFICACY OF OVERNIGHT INTRAGASTRIC FEEDING}

Table 2 shows the results obtained from the overnight intragastric feeding regimen in the six patients. Pre-breakfast hypoglycemia was prevented; venous $\mathrm{pH}$ remained normal; uric acid levels were diminished; and liver span decreased in most patients. Hypertriglyceridemia and lactic acidosis improved, but the values did not return to normal.

The most dramatic effect of the overnight intragastric infusion regimen was on long-term linear growth. Figure 1 shows the growth curves of the two patients treated over 4 years. Both patients had a rapid increase in linear growth after starting on the regimen and have continued to grow within the range of normal. Patient 3, begun on the regimen at 4 months of age, has grown steadily just below the 50th percentile of length for age. The three older children (patients 4,5 and 6) have shown improvement in biochemical abnormalities but not in linear growth. Recent studies in patients 4 and 5 suggest that their continued poor growth has been due to inadequate control of hypoglycemia and lactic acidosis during the daytime. Better control was achieved by increasing the frequency and carbohydrate content of their daytime feedings.

Table 1. Patients with glucose-6-phosphatase deficiency treated with nocturnal intragastric feeding

\begin{tabular}{clcc}
\hline Patient & Sex & Present age (yr) & Duration of therapy (yr) \\
\hline 1 & F & $108 / 12$ & $47 / 12$ \\
2 & M & $75 / 12$ & $41 / 12$ \\
3 & F & $32 / 12$ & $210 / 12$ \\
4 & F & $136 / 12$ & $16 / 12$ \\
5 & M & $156 / 12$ & $16 / 12$ \\
6 & F & $96 / 12$ & $10 / 12$ \\
\hline
\end{tabular}

\section{RELATIONSHIP OF BLOOD LACTATE TO DEXTROSE INFUSION RATE}

As shown in Table 2, overnight intragastric infusion of dextrose improved, but did not completely normalize blood lactate concentrations. To examine the relationship between lactate levels and the rate of dextrose infusion, blood lactate and plasma glucose concentrations were determined in the six patients at different rates of intragastric dextrose administration ranging from 3-34 $\mathrm{mg} / \mathrm{kg} / \mathrm{min}$. Figure $2 a$ shows an example of the relationship between blooc lactate and plasma glucose levels under various rates of dextrose infusion in one of the patients. Lower levels of plasma glucose were associated with higher levels of blood lactate $(r=-0.56, P<0.01)$. A similar significant inverse relation between blood lactate and plasma glucose levels was seen in four of the other five patients with $r$ values of -.43 to -.63 .

Figure $2 b$ shows the relationship between levels of blood lactate and the rate of intragastric dextrose infusion in patient 1 . Despite considerable variability in blood lactate values, rates of dextrose infusion less than $8 \mathrm{mg} / \mathrm{kg} / \mathrm{min}$ were associated with higher levels of blood lactate. Rates of dextrose infusions above $8-10 \mathrm{mg} / \mathrm{kg} /$ min did not appear to influence blood lactate concentrations. Analysis of the data in Figure $2 b$ by simple linear regression demonstrated a statistically significant inverse relation between blood lactate levels and the rate of dextrose infusion $(r=-.45, P$ $<0.05$ ). A similar inverse relationship was seen in a second patient $(r=f-.41, P<0.01)$, where dextrose infusion rates as low as 3 $\mathrm{mg} / \mathrm{kg} / \mathrm{min}$ were tried. No significant relationship was found in the remaining four patients in whom dextrose infusion rates were above $7 \mathrm{mg} / \mathrm{kg} / \mathrm{min}$.

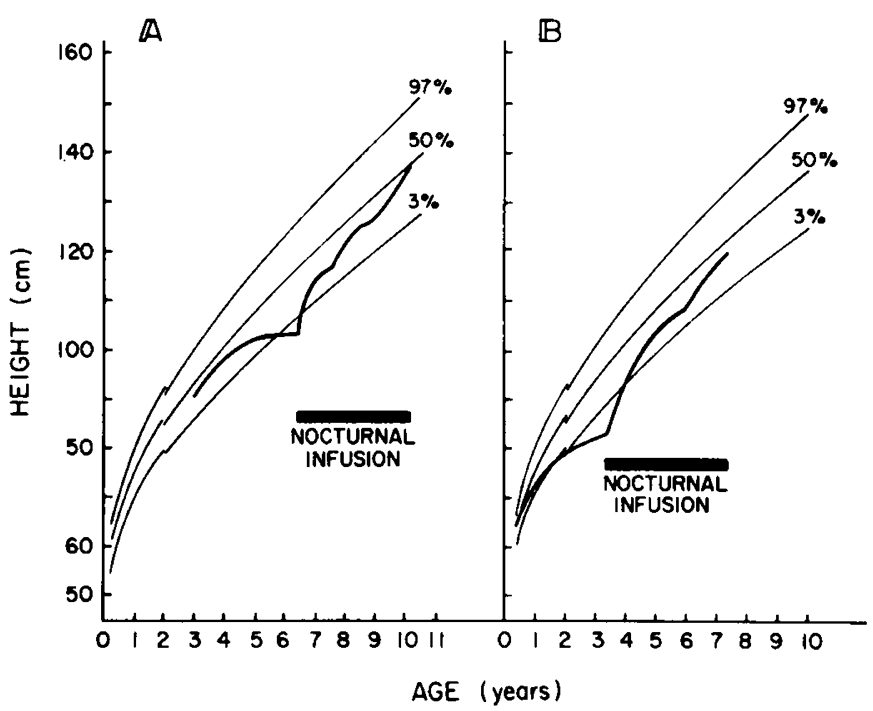

Fig. 1. Effect of treatment with nocturnal intragastric dextrose feeding on linear growth in two patients with glucose-6-phosphatase deficiency. Panel $A$ is patient 1 and panel $B$ is patient 2.

Table 2. Effect of nocturnal intragastric feeding in six children with glucose-6-phosphatase deficiency

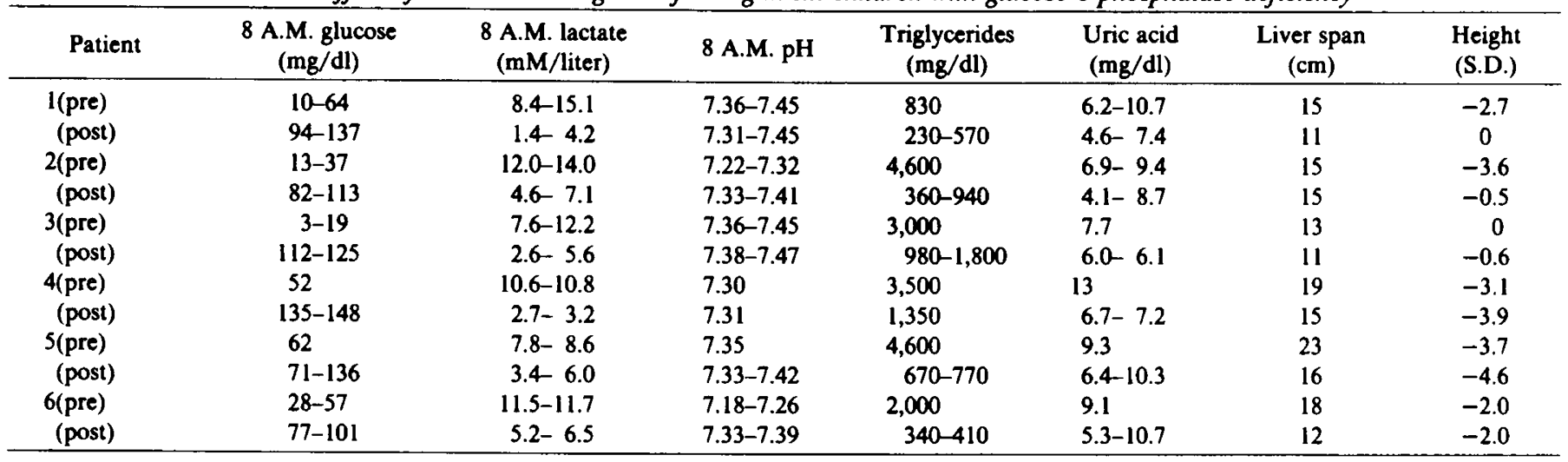



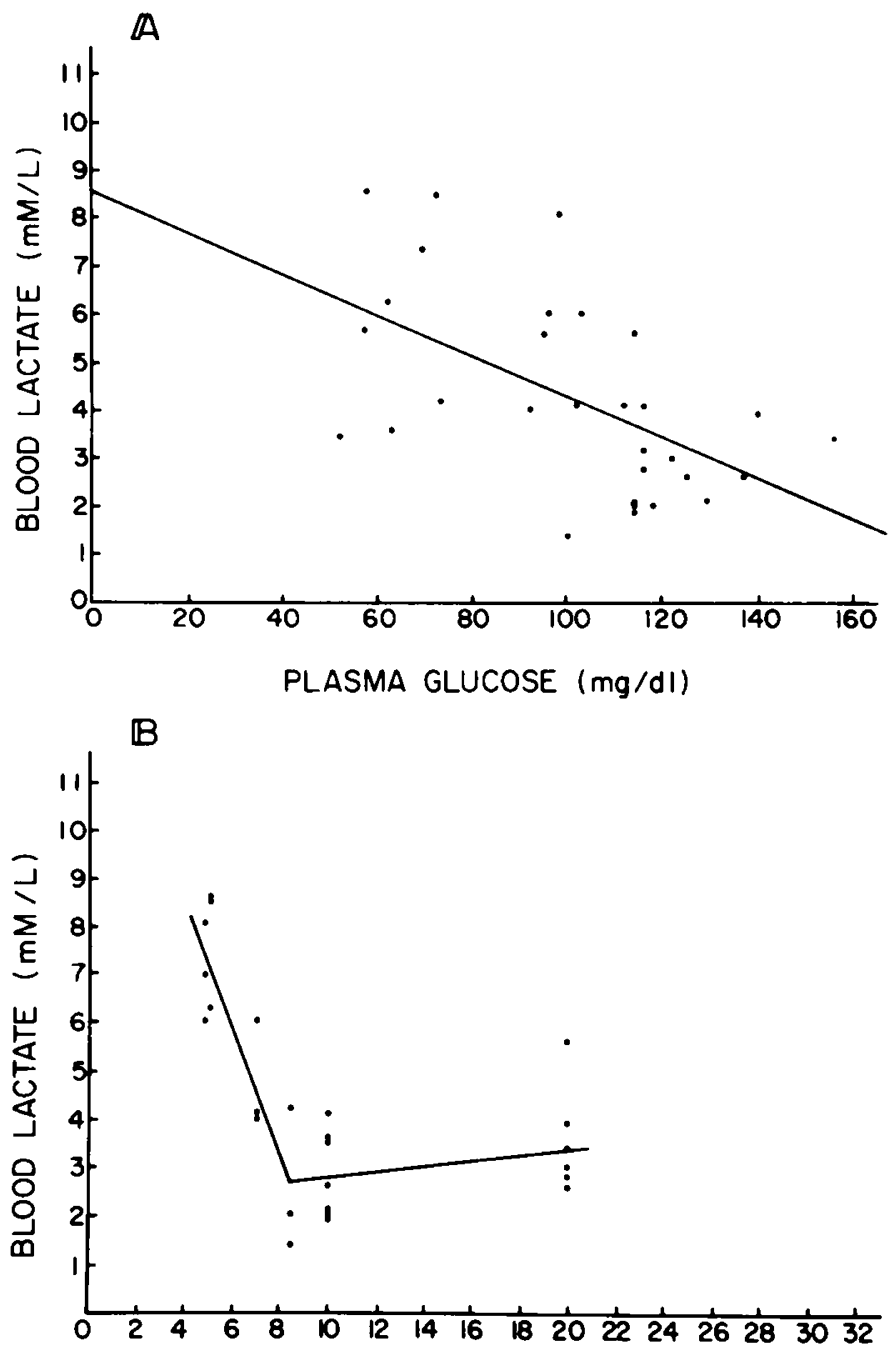

\section{DEXTROSE INFUSION RATE $(\mathrm{mg} / \mathrm{kg} / \mathrm{min}$ )}

Fig. 2. Relationship between blood lactate concentration and plasma glucose levels $(A)$ and rate of dextrose administration $(B)$ in patient 1 during continuous intragastric dextrose infusions.

Figure 3 shows the combined data on blood lactate and plasma glucose levels in the six patients at various rates of intragastric dextrose infusion. To correct for wide differences in weight, the dextrose infusion rates are expressed as a fraction of the glucose turnover rate found in normal children as reported by Bier, et al. (1). As shown, dextrose infusion at $100-150$ or $150-200 \%$ of estimated normal glucose production rates appeared to give the best control of blood lactate concentrations. Despite normal mean levels of plasma glucose, lower rates of dextrose infusion resulted in significantly higher blood lactate levels. Higher rates of dextrose infusions produced hyperglycemia, but no significant further reduction of blood lactate concentration.

\section{EFFECT OF COMPOSITION OF INTRAGASTRIC INFUSATE ON BLOOD} LACTATE

Previous reports have described treatment of patients with glucose-6-phosphatase deficiency using nocturnal intragastric infusions containing not only dextrose, but also other nutrients. Blood lactate levels in the patients reported from Vanderbilt (8, 15), where Vivonex was used, appeared to be lower than those seen in our patients treated with solutions containing only dextrose. In order to evaluate the effect of additional nutrients on control of hyperlactatemia, patients were monitored while receiving either dextrose alone or low (Cho-Free) or high (Vivonex) carbohydrate feedings at identical rates of glucose administration. The results are shown in Table 3 . In three patients, the additional calories provided by the low carbohydrate formula (Cho-Free) did not result in higher levels of plasma glucose and, in two of three, resulted in significantly higher levels of blood lactate than infusion of dextrose alone. In four patients, there was no significant improvement in control of blood lactate levels with the highcarbohydrate enteric feeding solution (Vivonex) compared with dextrose alone. In one patient, 5, levels of plasma glucose were significantly higher on Vivonex than on glucose infusions. However, similar degrees of hyperglycemia were observed with these two solutions in another patient, 4 , at identical rates of infusion.

\section{EFFECT OF ACUTE TERMINATION OF INTRAGASTRIC FEEDING ON CIRCULATING METABOLIC FUELS}

Previous reports $(15,13)$ have warned that patients with glucose6-phosphatase deficiency are more susceptible to developing symptomatic hypoglycemia after treatment is begun with the continuous intragastric feeding regimen. In order to examine whether intragastric feeding alters the response to fasting in these patients, levels of the major metabolic fuels were measured in patient 3 after a mixed meal prior to treatment, and compared with values seen after termination of intragastric dextrose infusion on two occasions (Table 4). The values obtained before treatment

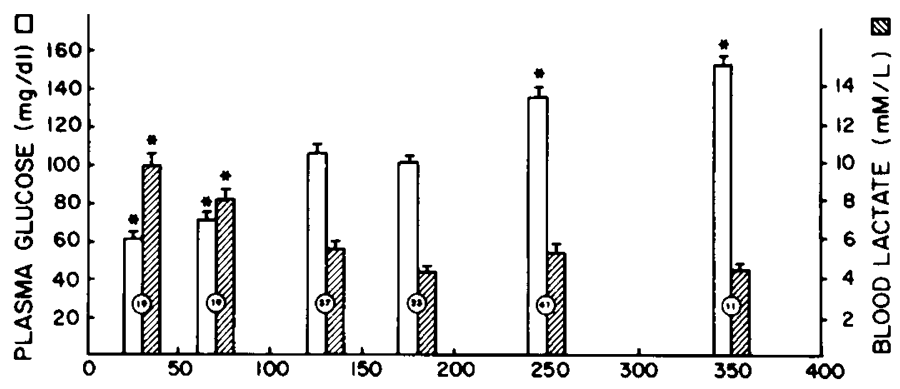

DEXTROSE INFUSION RATE (\% of normol glucose production rote)

Fig. 3. Relationship between plasma glucose and blood lactate concentrations and rate of intragastric dextrose infusion in patients with glucose6-phosphatase deficiency. Data show mean \pm S.E. of values obtained in studies on six patients. Rates of dextrose infusion are expressed as percent of hepatic glucose production in normal children (1). The number of values at each range of infusion rate is shown. Compared to dextrose infusion rates of $100-150 \%$ of "normal", $"=P<0.01$.

Table 3. Plasma glucose and blood lactate levels in patients with glucose-6-phosphatase deficiency during continuous intragastric feedings with Cho-Free or Vivonex, compared to dextrose alone (mean \pm S.E.)

\begin{tabular}{|c|c|c|c|c|}
\hline Patient & $\begin{array}{c}\text { Rate of } \\
\text { dextrose infusion } \\
\text { (\% of normal glucose } \\
\text { production) }\end{array}$ & $\begin{array}{l}\text { Infusate } \\
\text { (n) }\end{array}$ & $\begin{array}{l}\text { Glucose } \\
\text { (mg/dl) }\end{array}$ & $\begin{array}{c}\text { Lactate } \\
\text { (mM/liter) }\end{array}$ \\
\hline \multirow[t]{2}{*}{2} & $50-100$ & dextrose (3) & $76 \pm 9$ & $7.1 \pm 1.1$ \\
\hline & & Cho-Free (3) & $64 \pm 19$ & $9.1 \pm 2.0$ \\
\hline \multirow[t]{2}{*}{3} & $100-150$ & dextrose $(22)$ & $114 \pm 75$ & $6.0 \pm 0.6$ \\
\hline & & Cho-Free (5) & $99 \pm 0.6$ & $9.6 \pm 1.7^{1}$ \\
\hline \multirow[t]{2}{*}{3} & 150 & dextrose (4) & $109 \pm 6$ & $4.7 \pm 0.5$ \\
\hline & & Vivonex (4) & $94 \pm 8$ & $3.7 \pm 0.5$ \\
\hline \multirow[t]{2}{*}{6} & $150-200$ & dextrose (3) & $93 \pm 13$ & $6.0 \pm 0.2$ \\
\hline & & Vivonex (6) & $127 \pm 11$ & $5.0 \pm 0.5$ \\
\hline \multirow[t]{2}{*}{1} & $150-250$ & dextrose (6) & $86 \pm 11$ & $3.1 \pm 0.5$ \\
\hline & & Cho-Free (6) & $64 \pm 10$ & $6.7 \pm 0.9^{2}$ \\
\hline \multirow[t]{2}{*}{4} & $200-300$ & dextrose $(8)$ & $156 \pm 15$ & $6.8 \pm 1.2$ \\
\hline & & Vivonex (4) & $161 \pm 9.4$ & $4.9 \pm 0.9$ \\
\hline \multirow[t]{2}{*}{5} & $200-300$ & dextrose (6) & $99 \pm 6$ & $4.5 \pm 0.3$ \\
\hline & & Vivonex (4) & $143 \pm 13^{2}$ & $4.0 \pm 0.9$ \\
\hline
\end{tabular}

\footnotetext{
${ }^{1} P<0.05$.

${ }^{2} P<0.01$,
} 
Table 4. Circulating fuels during fasting in a patient with glucose6-phosphatase deficiency: (A) Following a meal, (B) and (C) After abrupt termination of intragastric dextrose infusion

\begin{tabular}{|c|c|c|c|c|c|c|}
\hline & & $\begin{array}{c}\text { Time } \\
\text { (hr) }\end{array}$ & $\begin{array}{l}\text { Glucose } \\
\text { (mg/dl) }\end{array}$ & $\begin{array}{c}\text { Lactate } \\
(\mathrm{mM} / \mathrm{li}- \\
\text { ter })\end{array}$ & $\begin{array}{c}\text { FFA } \\
(\mathrm{mM} / \\
\text { liter) }\end{array}$ & $\begin{array}{c}\text { BOB } \\
\text { (mM/ } \\
\text { liter) }\end{array}$ \\
\hline \multirow[t]{6}{*}{$A$. } & $\begin{array}{r}-1 \\
0\end{array}$ & (meal) & 46 & 7.7 & 0.78 & 0.16 \\
\hline & +1 & & 76 & & & \\
\hline & +2 & & 61 & 11.3 & & \\
\hline & +3 & & 55 & 11.3 & & 0.26 \\
\hline & +4 & & 32 & 11.1 & 0.88 & 0.26 \\
\hline & +5 & & 17 & 12.2 & 1.1 & 0.21 \\
\hline \multirow[t]{5}{*}{$B$. } & -12 & (on infusion) & 77 & 6.8 & & \\
\hline & -6 & & 117 & 2.9 & & \\
\hline & 0 & (infusion ends) & 115 & 5.6 & & \\
\hline & +1 & & 46 & 6.9 & 0.57 & 0.11 \\
\hline & +1.5 & & 21 & 8.0 & 0.56 & 0.15 \\
\hline \multirow[t]{4}{*}{ C. } & -4 & (on infusion) & 75 & 4.2 & 0.50 & 0.11 \\
\hline & 0 & (infusion ends) & 107 & 6.3 & 0.78 & 0.63 \\
\hline & +1 & & 37 & 5.8 & 0.75 & 0.24 \\
\hline & +1.5 & & 23 & 5.6 & 0.78 & 0.20 \\
\hline
\end{tabular}

demonstrate the typical features of disturbed fasting adaptation found with severe deficiency of glucose-6-phosphatase. Plasma glucose concentration remained in the normal range for only $3 \mathrm{~h}$ after the meal and then fell rapidly to $17 \mathrm{mg} / \mathrm{dl}$ at $5 \mathrm{~h}$. This severe hypoglycemia was associated with markedly elevated levels of blood lactate but no symptoms of central nervous system dysfunction. Levels of FFA were also raised at the time of hypoglycemia, but levels of BOB remained very low. This is in accord with the suggestion by several investigators $(2,6,10)$ that hepatic ketogenesis may be deficient in patients with glucose-6-phosphatase deficiency.

As shown in Table 4, the levels of plasma glucose fell rapidly after the intragastric dextrose infusion was stopped. On both occasions, plasma glucose was below $25 \mathrm{mg} / \mathrm{dl}$ within $90 \mathrm{~min}$. No symptoms of hypoglycemia were observed at this time. Blood lactate levels rose modestly in the first, but were unchanged during the second trial of stopping the infusion. Levels of free fatty acids were lower on both of these occasions than after the meal and failed to increase in response to the fall in plasma glucose concentration. These studies indicate that plasma glucose levels fall more rapidly when intragastric feeding is stopped than after a mixed meal. Hypoglycemia appears to develop before counter-regulatory responses can be mobilized.

\section{DISCUSSION}

The nocturnal intragastric infusion regimen described by Burr et al. (3) has been a major advance in the treatment of patients with glucose-6-phosphatase deficiency glycogen storage disease. Marked improvements have been documented in several of the biochemical and clinical abnormalities, with improved linear growth velocity being a particularly dramatic feature (Fig. 1).

In our six patients, the blood lactate and serum triglyceride concentrations demonstrated a decrease towards normal but remained elevated even after several years of therapy. In order to evaluate the persistent elevation of blood lactate levels, studies were carried out infusing dextrose at different rates, and also comparing dextrose alone with infusion of more complete formulas. The results of these experiments indicate that, while blood lactate concentrations are remarkably unstable in these patients, significant correlations do exist between blood lactate levels and both the plasma glucose level and the rate of dextrose administration. While low levels of plasma glucose are associated with marked elevations of blood lactate concentrations, "normal" plasma glucose values $(60-80 \mathrm{mg} / \mathrm{dl})$ may be associated with lactate levels which are still quite high (Fig. $2 a$ and 3). Blood lactate concentrations were minimized when dextrose infusion rates were equal to or slightly greater than the hepatic glucose production rate which would be seen in normal children (Fig. $2 b$ and 3). Infusion rates below that resulted in significant elevations of blood lactate levels, even at times of normoglycemia; infusion of dextrose at higher rates produced hyperglycemia but no further lowering of blood lactate concentrations.

The results from feedings other than dextrose alone (Table 3) suggest that it is the rate of glucose infusion which is the most important factor in lowering blood lactate concentrations. Additional calories in the form of fat and protein (Cho-Free with dextrose) did not prevent high blood lactate levels if given at rates which provided inadequate glucose. The use of Vivonex did not appear to give lower blood lactate levels than dextrose alone when infused at similar rates.

In practice, this has important implications. (1) Treatment aimed at maintenance of "normoglycemia" may not be sufficient. This is implied in the recent review of the Vanderbilt experience (9) where they advise that treatment should produce plasma glucose concentrations in the "high normal" range. (2) Monitoring of blood lactate concentrations is important in order to ensure that their levels are maximally lowered. (3) In order to achieve maximal lowering of blood lactate levels (and "high normal" glucose levels) intragastric infusion rates of glucose should be calculated to deliver approximately $100-150 \%$ of the normal hepatic glucose production rate [as derived from the data of Bier et al. (1)]. (4) Intragastric infusion of either Vivonex or Cho-Free with added dextrose did not result in significant improvements in plasma glucose or blood lactate concentrations when compared with simple dextrose infusions at the same rate. It is possible that adding amino acids to the infusate might have long-term benefits which were not detectable in the present short-term studies. Until such benefits are demonstrated, we feel that, because of its lower cost and similar efficacy, patients can be treated with dextrose alone in the overnight infusions.

Measurement of blood lactate levels to ensure that they are maximally lowered may be the most physiologic method of monitoring the efficacy of treatment in patients with glucose-6-phosphatase deficiency. Glucose-6-phosphatase catalyzes the final step in hepatic glucose production from both glycogenolysis and gluconeogenesis. By providing exogenous glucose, at a rate slightly greater than the liver would be called upon to provide during the overnight fast, there is no need for hepatic glucose production. The lowered blood lactate concentrations thus reflect the absence of signals to the liver for glucose production. The lowering of blood lactate concentrations also provides an amelioration of the chronic metabolic acidosis seen in these patients and which, possibly in association with the loss of base as urinary lactate (5), may play a role in the impairment of growth. Improvement in blood lactate concentration also appears to correlate with changes in the hormonal milieu which have been suggested by Slonim, $e t$ al. (15) to be associated with the improvement of growth velocity.

Patients with untreated glucose-6-phosphatase deficiency usually manifest very few symptoms referable to the central nervous system at times of hypoglycemia. However, with the continuous intragastric feeding regimen, these patients have been reported $(13,15)$ to be more symptomatic when hypoglycemic; indeed, one patient died because of hypoglycemia when the infusion tubing accidentally became disconnected during the night (13). Glucose absorption from the intestine ends more abruptly when the intragastric infusion is discontinued than after a mixed meal. The studies in our patient (Table 4) indicate that the levels of lactate, FFA and ketones are lower at the time of hypoglycemia following cessation of the intragastric feeding than in untreated patients with a similar degree of hypoglycemia. It is not clear whether or not these low levels contribute significantly to the symptoms seen at the time of hypoglycemia. It is possible that the low levels reflect a difficulty in mounting a counter-regulatory response to the rapidly falling plasma glucose level following abrupt cessation of the intragastric infusion. The clinical corollary, of course, is that patients must be fed within 15-30 min after the end of the nasogastric infusion. 
The present study confirms previous reports that hypertriglyceridemia is only partly corrected with the treatment of glucose-6phosphatase deficiency by nocturnal intragastric feedings (Table 2). Fernandez et al. (5) have suggested that this is due to decreased triglyceride clearance, since lipoprotein lipase activity remained low in their treated patients. However, it is also possible that elevated levels of gluconeogenic precursors play a role in the abnormalities of lipid metabolism seen in both treated and untreated patients. McGarry et al. (14) have shown that malonyl$\mathrm{CoA}$, an intermediate in fatty acid synthesis from pyruvate, inhibits the external mitochondrial carnitine-acyl-transferase, the regulatory step in fatty acid oxidation. In untreated patients, high levels of lactate and pyruvate might prevent an appropriate fall in hepatic malonyl-CoA levels during fasting, and impair the oxidation of fatty acid delivered to the liver from adipose tissue. This would account for the massive accumulation of fat in the liver in untreated patients and the impairment in ketogenesis suggested by other workers $(2,6,10)$ and our own data (Table 4). In treated patients, persistent elevations of lactate and pyruvate may support excessive hepatic fatty acid synthesis and triglyceride production. That is, triglyceride formation may represent a major route for disposal of pyruvate from lactate and amino acids when glucose synthesis is blocked by deficiency of glucose-6-phosphatase. If this formulation is correct, it may not be possible to lower plasma triglyceride concentrations into the normal range using only continuous nocturnal intragastric infusions and frequent daytime feedings.

\section{REFERENCES AND NOTES}

1. Bier, D. M., Leake, R. D., Haymond, M. W., Arnold, K. J., Gruenke, L. D., Sperling, M. A., and Kipnis, D. M.: Measurement of "true" glucose production rates in infancy and childhood with 6,6 dideuteroglucose. Diabetes, 26: 1016 (1977).

2. Binkiewicz, A., and Senior, B.: Decreased ketogenesis in von Gierke's disease (Type I glycogenosis). J. Pediatr., 83: 973 (1973).

3. Burr, I. M., O'Neill, J. A., Karzon, D. T., Howard, L. J., and Greene, H. L. Comparison of the effects of total parenteral nutrition, continuous intragastric feeding, and portacaval shunt on a patient with Type 1 glycogen storage disease. J. Pediatr., 85: 792 (1974).

4. Ehrlich, R. M., Robinson, B. H., Freedman, M. H., and Howard, N. J.: Nocturnal intragastric infusion of glucose in management of defective gluconeogenosis with hypoglycemia. Am. J. Dis. Child, I32: 241 (1978).

5. Fernandes, J., Jansen, H., and Jansen, T. C.: Nocturnal gastric drip feeding in glucose-6-phosphatase deficient children. Pediatr. Res., 13: 25 (1979).

6. Fernandes, J., and Pikaar, N. A.: Ketosis in hepatic glycogenosis. Arch. Dis. Child., 47: 41 (1972).

7. Greene, H. L., Slonim, A. E., Burr, I. M., and Moran, J. R.: Type I glycogen storage disease: five years of management with nocturnal intragastric feeding. J. Pediatr., 96: 590 (1980).

8. Greene, H. L., Slonim, A. E., O'Neill, J. A., and Burr. I. M.: Continuous nocturnal intragastric feeding for management of type I glycogen-storage disease. N. Engl. J. Med., 294: 423 (1976).

9. Greene, H. L., Slonim, A. E., and Burr, I. M.: Type I glycogen storage disease: a metabolic basis for advances in treatment. Adv. in Pediatr., 26: 63 (1979).

10. Havel, J. R., Balasse, E. O., Williams, H. E., Kane, J. P., and Segel, N.: Splanchnic metabolism and von Gierke's disease (Glycogenosis Type 1). Trans. Assoc. Amer. Phys., 82: 305 (1969).

11. Howell, R. R.: The Glycogen Storage Diseases in the Metabolic Basis of Inherited Disease. In: Stanbury, J. B., Wyngaarden, J. B., Fredrickson. D. S. (Eds), pp 143-146 McGraw Hill, New York (1978).

12. Kelch, R. C., and Oliver, W. J.: Studies on dietary correction of metabolic abnormalities in hepatorenal glycogenosis. Pediatr. Res., 3: 160 (1969).

13. Leonard, J. V., and Dunger, D. B.: Hypoglycemia complicating feeding regimens for glycogen-storage disease. Lancet, 2: 203 (1978).

14. McGarry, J. D., Mannaerts, G. P., and Foster, D. W.: A possible role for malonylCoA in the regulation of hepatic fatty acid oxidation and ketogenesis. J. Clin. Invest., 60: 265 (1977).

15. Slonim, A. E., Lacy, W. W., Terry, A., Greene, H. L., and Burr, I. M.: Nocturnal intragastric therapy in type I glycogen storage disease: effect on hormonal and amino acid metabolism. Metabolism, 28: 707 (1979).

16. Stanley, C. A., and Baker, L.: Hyperinsulinism in infancy: diagnosis by demonstration of abnormal response to fasting hypoglycemia. Pediatr., 57: 702 (1976).

17. The authors thank the nurses of the Clinical Studies Unit for providing excellent care of the patients in this study, Joseph Egler for expert technical assistance and Sharon Billups and Patti Kozinski for help in preparing this manuscript.

18. Requests for reprints should be addressed to: Dr. Charles A. Stanley, Division of Endocrinology/Diabetes, Children's Hospital of Philadelphia, One Children's Ctr., Philadelphia, PA 19104.

19. This research was supported in part by NIH grants AM-13518, RR240, AM19525 and a Robert Wood Johnson Foundation Fellowship (J.L.M.).

20. Received for publication February 13, 1981.

21. Accepted for publication June 1, 1981. 